\title{
Long-term ischaemic and bleeding outcomes after primary percutaneous coronary intervention for ST-elevation myocardial infarction in the elderly
}

\author{
Bimmer E.P.M. Claessen • Wouter J. Kikkert • Loes P. Hoebers • \\ Hassina Bahadurzada • Marije M. Vis - Jan Baan - Karel T. Koch • \\ Robbert J. de Winter · Jan G.P. Tijssen · Jan J. Piek · José P.S. Henriques
}

Published online: 11 August 2015

(C) The Author(s) 2015. This article is published with open access at Springerlink.com

\begin{abstract}
Background The population is ageing rapidly and the proportion of patients aged $\geq 80$ years undergoing primary percutaneous coronary intervention (PCI) is rising, but clinical trials have primarily been performed in younger patients. Methods Patients undergoing primary PCI between 2003 and 2008 were subdivided into 3 groups: $<60,60-79$, and $\geq 80$ years. Endpoints at 3 -year follow-up included all-cause mortality, recurrent myocardial infarction (reMI), stent thrombosis, target lesion revascularisation (TLR), bleeding (BARC bleeding $\geq 3$ ), stroke, and major adverse cardiovascular events (MACE, a composite of cardiac mortality, reMI, stroke and TLR).

Results 2002 patients with ST-segment elevation myocardial infarction (STEMI) were included, 885 (44.2\%) aged $<60,921(46.0 \%) 60-79$, and $196(9.7 \%) \geq 80$ years. Comorbidities such as diabetes mellitus, prior stroke, malignant disease, anaemia, and chronic kidney disease were more prevalent in patients $\geq 80$ years. The incidence of both ischaemic and bleeding events strongly increased with age. Age $\geq 80$ years was an independent predictor of mortality (HR 2.56, 95\% CI1.69-3.87, $p<0.001$ ), a borderline nonsignificant predictor of overall bleeding (HR $1.38,95 \% \mathrm{CI}$ $0.95-2.00, p=0.088$ ), and a significant predictor of non-access site bleeding (HR 2.26, 95\%CI 1.46-3.51, $p<0.001$ ).
\end{abstract}

B. E. Claessen and W. J. Kikkert authors contributed equally.

B. E.P.M. Claessen $(\bowtie) \cdot$ W. J. Kikkert · L. P. Hoebers ·

H. Bahadurzada · M. M. Vis · J. Baan · K. T. Koch ·

R. J. de Winter · J. G.P. Tijssen · J. J. Piek · J. P.S. Henriques

Department of Cardiology, B2-115,

Academic Medical Center - University of Amsterdam,

Meibergdreef 9,

1105 Amsterdam, AZ, The Netherlands

e-mail: b.e.claessen@amc.uva.nl
Conclusion Patients $\geq 80$ years experienced high rates of ischaemic and bleeding complications; especially in this high-risk patient group individualised therapy is needed to optimise clinical outcomes.

Keywords Primary percutaneous coronary intervention . Acute myocardial infarction $\cdot$ Stent $\cdot$ Elderly

\section{Introduction}

For patients with ST-segment elevation myocardial infarction (STEMI), primary percutaneous coronary intervention (PCI) is the preferred therapy $[1,2]$. Guidelines have been composed to suggest optimal antithrombotic and antiplatelet regimens in order to prevent new ischaemic events while balancing the risk of bleeding events [2,3]. However, due to the frailty that comes with advanced age, the higher prevalence of diverse comorbidities in the elderly, and the fact that large clinical trials often list age $\geq 80$ years as an exclusion criterion, it is currently unclear if following guideline recommendations will also result in acceptable outcomes regarding ischaemic and bleeding endpoints in elderly patients undergoing primary PCI.

The mean age of the population in the developed world is increasing rapidly [4]. In the European Union, the proportion of people aged $\geq 80$ years is expected to triple from 4.8 to $12.0 \%$ between 2010 and 2060 [5]. An increase in the absolute and relative number of octogenarians undergoing primary PCI has been described [6]. A small number of observational studies have reported mortality rates after primary PCI for STEMI in patients aged $\geq 80$ years [6-9]. However, there is a paucity of data on the long-term occurrence of recurrent ischaemic events and bleeding in the elderly after primary PCI. 
We therefore investigated a cohort of STEMI patients undergoing primary PCI in a large tertiary care centre to provide more insight into ischaemic and bleeding outcomes after primary PCI for STEMI in patients aged 80 years and older.

\section{Methods}

Data analysed in this study were obtained from STEMI patients who were accepted for primary PCI at the Academic Medical Center-University of Amsterdam between 1 January 2003, and 31 July 2008. The study complied with the Declaration of Helsinki, and the local ethics committee approved the study protocol. In general, patients qualified for primary PCI if they had typical ischaemic chest pain and at least $1 \mathrm{~mm} \mathrm{ST-segment} \mathrm{elevation} \mathrm{in} \mathrm{two} \mathrm{or} \mathrm{more} \mathrm{con-}$ tiguous leads or a new left bundle branch block (LBBB). The primary PCI and adjunctive pharmacological treatment were performed according to the American College of Cardiology/American Heart Association and the European Society of Cardiology (ESC) guidelines. Patients received a standard 300-600 mg clopidogrel loading dose. If a coronary stent was implanted, clopidogrel was prescribed for at least one month to patients with a bare metal stent (BMS) and for 6 to 12 months to patients with a drug-eluting stent (DES).Patients were routinely pretreated with $300-500 \mathrm{mg}$ aspirin and $5000 \mathrm{IU}$ unfractionated heparin (UFH). Glycoprotein IIb/IIIa inhibitors (GPIs) were used in a bail-out setting at the discretion of the operator.

A detailed description of the study protocol has been previously published [10-12]. In short, procedural and angiographic data were prospectively collected by interventional cardiologists and specialised nurses in a dedicated database. We obtained follow-up of clinical outcome, including recurrent MI, stroke, stent thrombosis and bleeding, by reviewing inpatient and outpatient charts in the tertiary PCI centre and referring hospitals between 2011 and 2012 for consecutive STEMI patients with available activated partial thromboplastin time (aPTT) measurements in the context of a study designed to investigate the relationship between aPTT and clinical outcome in STEMI patients treated with primary PCI. Follow-up of clinical events was censored at the date of chart review. Patients whose whereabouts were unknown were considered lost to follow-up from the date of last known medical contact. Follow-up information regarding vital status was obtained from computerised, long-term mortality records from the National Death Index between 1 December 2012 and 30 April 2012.

\section{Study design}

The study cohort consisted of all STEMI patients included in our study database who were alive at the end of the pro- cedure. Clinical outcomes included major adverse cardiac events (MACE; a composite of cardiac death, recurrent myocardial infarction (MI), stroke and target lesion revascularisation (TLR)), all-cause, cardiac and non-cardiac mortality, recurrent MI, ischaemic and haemorrhagic stroke, stent thrombosis, TLR and bleeding. Cardiac death, recurrent MI, stent thrombosis and TLR were defined according to the Academic Research Consortium (ARC) criteria [13]. Bleeding complications were defined according to the Bleeding Academic Research Consortium (BARC) bleeding classification and the Thrombolysis In Myocardial Infarction bleeding classification [14, 15] Stroke was defined as an irreversible neurological deficit, as classified by the treating neurologist, on the basis of supporting information, including brain images and neurological evaluation.

\section{Statistical analysis}

Normally distributed continuous variables are reported as the mean with standard deviation and compared with the one-way ANOVA test, skewed distributed variables are presented as the median with interquartile range (IQR) and compared with the Kruskal-Wallis test. Categorical variables are presented as proportions and compared with the $\chi^{2}$ test for trend. Event rates of clinical outcomes were estimated using Kaplan-Meier analyses. Missing covariate data were imputed with the use of multiple imputations and were assumed to be missing at random. We performed 10 imputations using the Markov Chain Monte Carlo method. Cox proportional hazards models were first fit separately in the 10 imputed datasets and subsequently pooled according to Rubin's protocol [16]. Multivariable Cox proportional hazards regression was used in the following manner: first, univariable hazard ratios were calculated for all variables in Tables 1 and 2. Then, multivariable backward stepwise Cox regression models were constructed using variables with $p<0.10$ in univariate analysis as candidate variables. Entry and exit criteria were set at $p=0.10$.

In order to plot the graphic relationships between age and probability of death, MACE, BARC type $\geq 3$ bleeding and stroke at 3 years, we calculated the predicted probability of these events by incorporating the coefficients returned by the unadjusted and adjusted Cox models in the following equation:

$$
S(t)=e^{\left(-H_{o}(t) *\left(x_{1} \beta_{1}+x_{2} \beta_{2}+\ldots x_{k} \beta_{k}\right)\right)}
$$

Where $\mathrm{S}(\mathrm{t})$ is the predicted event-free survival at timepoint $\mathrm{t}, \mathrm{H}_{0}(\mathrm{t})$ is the baseline hazard at time $\mathrm{t}, \mathrm{x}_{1,2, \ldots \mathrm{k}}$ is the vector of the covariates, and $\beta_{1,2, \ldots \mathrm{k}}$ the coefficients determined from the Cox regression models. The risk of adverse events was 
calculated as $1 \mathrm{~min} \mathrm{~S}(\mathrm{t})$. For the present analysis, we assumed ' $t$ ' to be 1095 days for all patients. The predicted probabilities were pooled over the imputed datasets utilising Rubin's protocol after applying the logit transformation to the predicted probabilities [17]. The relationship between age and the probability of death, MACE, stroke and bleeding were plotted graphically using a model fitting approach involving cubic polynomials (splines) as previously employed by the GUSTO-I (Global Utilization of Streptokinase and Tissue Plasminogen Activator for Occluded Coronary Arteries) trial investigators [18]. All analyses were performed with Statistical Package for Social Sciences software (SPSS version 19.0, Chicago, Illinois).

\section{Results}

Of 2009 STEMI patients recorded in our database who were treated with primary PCI in our institution between 1 January 2003 and 31 July 2008, 2002 were alive at the end of the procedure. Median follow-up duration in this cohort of patients was 4.9 years (IQR 3.4-6.4 years). A total of 196 patients were aged 80 years or older $(9.7 \%), 921$ patients were aged $60-79$ years $(46.0 \%)$, and 885 patients were aged $<60$ years $(44.2 \%)$.

Baseline characteristics are shown in Table 1. The proportion of female patients increased with age; among patients aged $\geq 80$ years the majority of patients were female. Patients aged $\geq 80$ years were more likely to have diabetes mellitus, prior coronary artery bypass graft surgery (CABG), a history of stroke or transient ischaemic event, peripheral artery disease and malignant disease compared with younger patients. Moreover, they more often had anaemia and an estimated creatinine clearance of $<60 \mathrm{ml} /$ $\min / 1.73 \mathrm{~m}^{2}$. On the other hand, patients aged $\geq 80$ years were less likely to be smokers, less often had a positive family history of coronary artery disease and had lower rates of hypercholesterolaemia at admission compared with younger patients.

Angiographic and procedural characteristics are shown in Table 2. Patients aged $\geq 80$ years had longer ischaemic times, a higher prevalence of cardiogenic shock at admission, lower rates of post-procedural TIMI 3 flow, a higher rate of coronary calcification, multivessel disease and chronic total occlusions in non-infarct related vessels compared with younger patients.

Figure 1 displays a Kaplan-Meier time-to-event curve for MACE. Figure 2 displays Kaplan-Meier time-to-event curves for recurrent MI, mortality, BARC bleeding $\geq 3$, and stroke. Table 3 shows Kaplan-Meier estimates of the incidence of adverse events at 3-year follow-up. Rates of MACE, mortality, recurrent MI, stroke and BARC bleeding $\geq 3$ increased with age. Conversely, rates of stent thrombo-

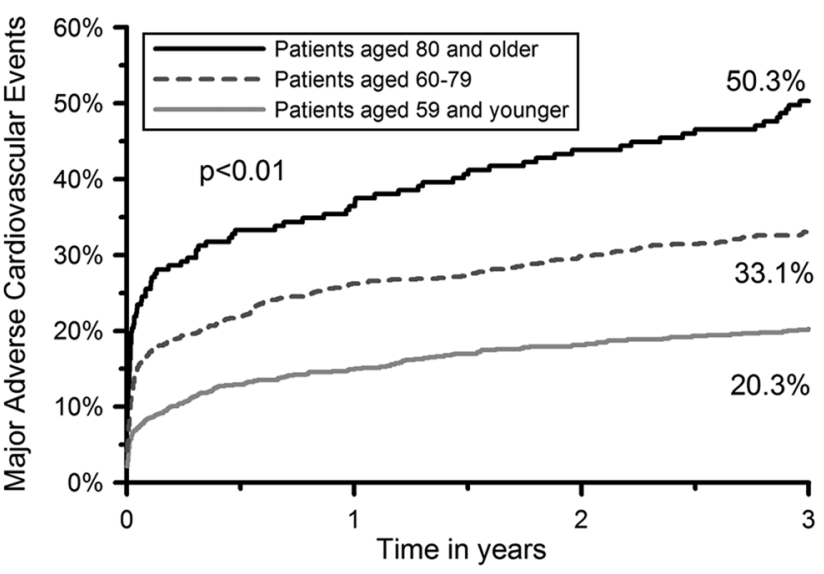

Fig. 1 Kaplan-Meier curve for major adverse cardiac events

sis and target lesion revascularisation decreased non-significantly with age.

Table 1 of the Supplementary Appendix shows the results of multivariable Cox regression analyses for 3-year clinical outcomes. Relative to patients aged $<60$ years, patients aged $60-79$ years and those aged $\geq 80$ years were found to have an increased risk of cardiac and non-cardiac mortality, stroke and non-access site bleeding after correction for confounding variables. In addition, age 60-79 years and age $\geq 80$ years was associated with a borderline non-significant increase in risk of BARC type $\geq 3$ bleeding. On the other hand, age $\geq 80$ years was not an independent predictor of MACE. Detailed multivariable models are shown in the Supplementary Appendix.

Age as a continuous variable was an independent predictor of all-cause mortality (1.43 per 10 year increase; 95\% CI 1.27-1.61; $p<0.001$ ), cardiac mortality (HR 1.34; 95\% CI 1.17-1.53, $p<0.001$ ), non-cardiac mortality (HR $1.55 ; 95 \%$ CI $1.24-1.95, p<0.001$ ), stroke (HR 1.46; $95 \%$ CI $1.18-1.80 ; p<0.001$ ) and BARC type $\geq 3$ bleeding (HR $1.15 ; 95 \%$ CI $1.04-1.26 ; p=0.006$ ), but not of MACE (HR 1.06 ; $95 \%$ CI $0.98-1.16 ; p=0.17$ ), or recurrent MI (HR 1.04; $95 \%$ CI $0.93-1.17, p=0.49$ ) (Supplementary Appendix, Table 2). Figure 3 shows a graphic representation of the unadjusted (left panel) and adjusted (right panel) relation between age as a continuous variable and the hazard of MACE, mortality, BARC bleeding $\geq 3$, and stroke up to 3 -year follow-up.

\section{Discussion}

The current study extends previous reports by providing insight into the occurrence of a large number of clinically relevant endpoints in up to 3 years of follow-up in patients undergoing primary PCI according to age. We found higher rates of ischaemic and haemorrhagic endpoints with increas- 
Fig. 2 Kaplan-Meier curve for recurrent myocardial infarction, mortality, BARC bleeding $\geq 3$, and stroke. $B A R C$ bleeding academic research consortium
Fig. 3 Probability of 3-year mortality, major adverse cardiac events, stroke, and BARC bleeding $\geq 3$ according to age. $M A C E$ major adverse cardiovascular event, $B A R C$ bleeding academic research consortium
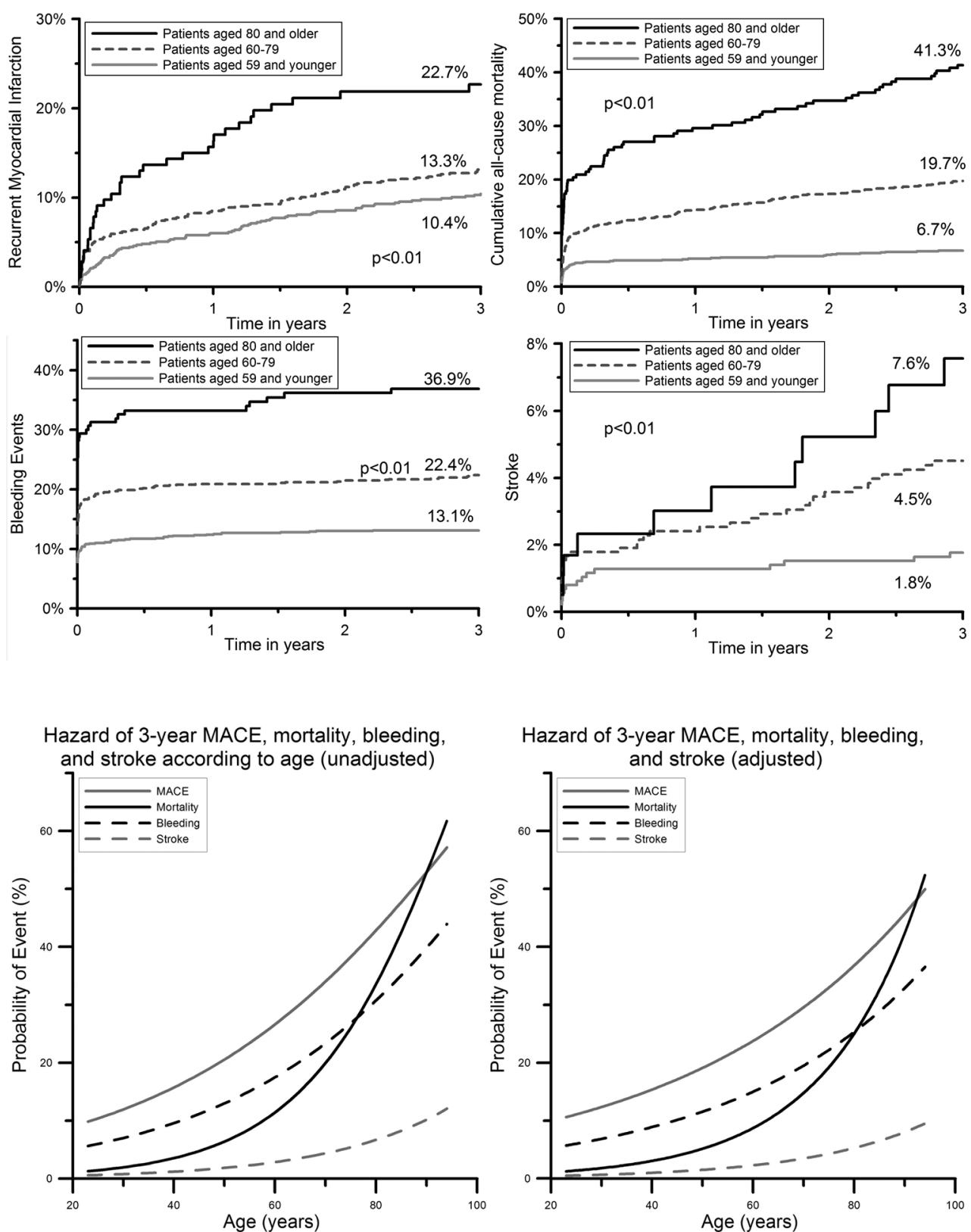

Hazard of 3-year MACE, mortality, bleeding,

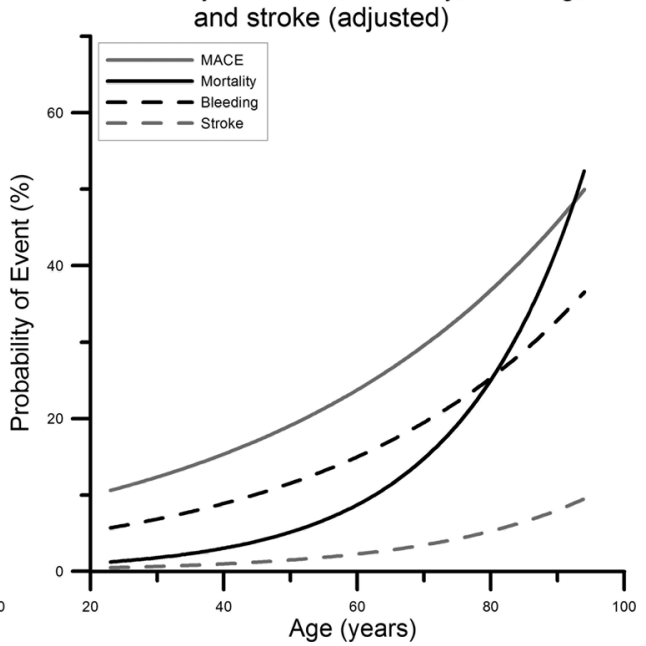

ing age, illustrating the frailty of this rapidly growing highrisk patient group.

In a recent large registry, Velders et al. found an increased risk of early mortality and heart failure in patients aged $\geq 80$ as compared with patients younger than 80 [8]. However, among the elderly patients who survived the first month(s), the prognosis was similar compared with their younger counterparts. This indicates that the difference in mortality between patients aged $\geq 80$ and their younger counterparts can be explained by the direct adverse consequence of the STEMI itself, such as arrhythmia, heart failure, mechanical complications and procedural complications such as bleeding. Interestingly, consistent with previous studies, we found that elderly patients were less likely to develop stent thrombosis or to undergo target lesion revascularisation $[19,20]$. The fact that the rate of recurrent MI was higher among elderly patients suggests that recurrent $\mathrm{MI}$ in elderly patients is the result of plaque rupture of non-target lesions, and is likely explained by the more extensive atherosclerosis among the elderly.

The incidence of both access site and non-access site bleeding strongly increased with age in this study. Wellknown risk factors for bleeding such as female gender and chronic kidney disease were more prevalent in the elderly [21]. Moreover, advanced age is associated with increased vascular fragility and calcification, which are known risk factors for access site bleeding $[22,23]$. In the current study, age $\geq 80$ years was independently associated with non- 
access site bleeding, an important finding given the fact that especially non-access site bleeding is associated with adverse outcome [21, 24]. In a pooled patient-level analysis involving 14,180 patients recruited in 7 randomised trials PCI treatment with a GP IIb/IIIa inhibitor was associated with a significant $27 \%$ higher risk of non-access site bleeding [25]. A large observational study in 10,469 patients aged $\geq 80$ years included in the CathPCI registry suggests that bleeding avoidance strategies such as radial access and use of vascular closure devices were independently associated with lower bleeding [26]. A number of recommendations to reduce bleeding complications in the elderly may be hypothesised: (1) the radial approach would seem to be the preferred method of vascular access in patients aged $\geq 80$ years; (2) Routine proton pump inhibition might reduce gastrointestinal bleeding in patients aged $\geq 80$ years; and (3) use of GP IIb/IIIa inhibitors in the elderly may lead to increased bleeding and should be avoided where possible.

By observing the Kaplan-Meier curves for bleeding, recurrent MI, and stroke, it appears that bleeding predominantly occurs during the first month after primary PCI, whereas the incidence of recurrent MI and stroke (of the ischaemic type in the vast majority of patients) continues to increase during the 3-year follow-up period. Studies investigating novel P2Y12 inhibitors such as prasugrel and ticagrelor have reported superior outcomes compared with clopidogrel with regards to ischaemic events, at a cost of increased bleeding events $[27,28]$. No studies investigating strategies to reduce MI and stroke after primary PCI for STEMI in patients $\geq 80$ years have been conducted yet. It is therefore currently still unclear whether there is a role for novel P2Y12 inhibitors in this population.

Recently, a small number of observational studies have reported results of primary PCI for STEMI in patients aged $\geq 80$ years, showing 30 -day mortality rates of around $28 \%$ [6-8]. The high rates of mortality in patients aged $\geq 80$ years can largely be explained by a higher burden of comorbidities, a higher rate of haemodynamic instability during primary PCI, and high rates of bleeding and recurrent MI as shown in the current study. While acknowledging that advanced age itself is an important and unmodifiable predictor of mortality, a strategy aimed at reducing early bleeding complications, and late ischaemic complications might potentially lead to improved mortality in patients aged $\geq 80$ years. Recent developments in primary PCI associated with superior outcomes such as the radial rather than the femoral approach becoming the default vascular access strategy in primary PCI, and the introduction of more effective antiplatelet agents such as prasugrel and ticagrelor instead of clopidogrel may have the greatest absolute benefit in patients aged $\geq 80$ years, as these patients also have the highest absolute event rates of adverse events [29]. Nonetheless, the novel P2Y12 inhibitors that are relatively more effective in inhibiting platelet aggregation compared with clopidogrel may also cause more bleeding events in the elderly, potentially offsetting the reduction in ischaemic events observed in randomised controlled trials. Ideally, this should be investigated in a randomised clinical trial enrolling STEMI patients aged $\geq 80$ years.

\section{Limitations}

A number of limitations of the present study deserve mentioning. First, only a relatively small cohort of patients aged $\geq 80$ years were included in the present analysis. Nonetheless, this is the largest cohort of very elderly patients to date with detailed reporting of a multitude of 3-year ischaemic and bleeding events. Moreover, due to the high event rates, this cohort was sufficiently powered to conduct multivariable analyses. Finally, as this was an observational study we can only speculate about treatment strategies to improve the clinical outcome of very elderly patients undergoing primary PCI.

\section{Conclusion}

Patients $\geq 80$ years experienced high rates of ischaemic and bleeding complications, especially in this high-risk patient group individualised therapy is needed to optimise clinical outcomes.

Funding source This work was supported by The Nuts OHRA Foundation, the Netherlands [SNO-T-0702-61]

Acknowledgements The authors greatly acknowledge the staff of the Departments of Cardiology of the following hospitals for their assistance during data collection (alphabetical order): BovenIJ Hospital, Bronovo, Diakonessenhuis Utrecht, Flevohospital, Gelre Hospitals, Gemini Hospital, HagaHospital, Kennemer Gasthuis, MC Zuiderzee, Meander Medical Centre, Medical Centre Alkmaar, Medical Centre Haaglanden, Onze Lieve Vrouwe Gasthuis, Red CrossHospital Beverwijk, Sint Lucas Andreas Hospital, Slotervaarthospital, Spaarne Hospital, St. Antonius Hospital, Tergooi Hospitals, VU University Medical Centrum, Westfriesgasthuis, Amstelland Hospital, Zuwe Hofpoort Hospital.

Conflicts of interest There are no conflicts of interest to declare.

Open Access This article is distributed under the terms of the Creative Commons Attribution License which permits any use, distribution, and reproduction in any medium, provided the original author(s) and source are credited.

\section{References}

1. Keeley EC, Boura JA, Grines CL. Primary angioplasty versus intravenous thrombolytic therapy for acute myocardial infarction: a quantitative review of 23 randomised trials. Lancet. 2003;361:13-20. 
2. Levine GN, Bates ER, Blankenship JC, et al. 2011 ACCF/AHA/ SCAI guideline for percutaneous coronary intervention. A report of the American College of Cardiology Foundation/American Heart Association Task Force on Practice Guidelines and the Society for Cardiovascular Angiography and Interventions. J Am Coll Cardiol. 2011;58:e44-122.

3. Steg PG, James SK, Atar D, et al. ESC Guidelines for the management of acute myocardial infarction in patients presenting with ST-segment elevation. Eur Heart J. 2012;33:2569-619.

4. United Nations Department of Social And Economic Affairs. World Population Ageing 1950-2050. 2002. www.unorg/esa/population/publications/worldageing19502050/. Accessed 16. Feb. 2015

5. European Commission. The 2012 ageing report. eceuropaeu/economy finance/publications/european economy/2012/ee-2012-2 enpdf 2012. Accessed 09. Apr. 2014.

6. Claessen BE, Kikkert WJ, Engstrom AE, et al. Primary percutaneous coronary intervention for ST elevation myocardial infarction in octogenarians: trends and outcomes. Heart. 2010;96:843-7.

7. Antonsen L, Jensen LO, Terkelsen CJ, et al. Outcomes after primary percutaneous coronary intervention in octogenarians and nonagenarians with ST-segment elevation myocardial infarction: from the Western Denmark heart registry. Catheter Cardiovasc Interv. 2013;81:912-9.

8. Velders MA, James SK, Libungan B, et al. Prognosis of elderly patients with ST-elevation myocardial infarction treated with primary percutaneous coronary intervention in 2001 to 2011: a report from the Swedish Coronary Angiography and Angioplasty Registry (SCAAR) registry. Am Heart J. 2014;167:666-73.

9. Moonen LA, van 't Veer M, Pijls NH. Procedural and long-term outcome of primary percutaneous coronary intervention in octogenarians. Neth Heart J. 2010;18:129-34.

10. Kikkert WJ, van Nes SH, Lieve KV, et al. Prognostic value of post-procedural aPTT in patients with ST-elevation myocardial infarction treated with primary PCI. Thromb Haemost. 2013;109:961-70.

11. Kikkert WJ, Zwinderman AH, Vis MM, et al. Timing of mortality after severe bleeding and recurrent myocardial infarction in patients with ST-segment-elevation myocardial infarction. Circ Cardiovasc Interv. 2013;6:391-8.

12. Kikkert WJ, van Geloven N, van der Laan MH, et al. The prognostic value of Bleeding Academic Research Consortium (BARC) defined bleeding complications in ST-segment elevation myocardial infarction: a comparison with the Thrombolysis In Myocardial Infarction (TIMI), Global Utilization of Streptokinase and Tissue Plasminogen Activator for Occluded Arteries (GUSTO) and International Society of Thrombosis and Haemostasis (ISTH) bleeding classifications. J Am Coll Cardiol. 2014;63:1866-75

13. Cutlip DE, Windecker S, Mehran R, et al. Clinical end points in coronary stent trials. Circulation. 2007;115:2344-51.

14. Mehran R, Rao SV, Bhatt DL, et al. Standardized bleeding definitions for cardiovascular clinical trials: a consensus report from the Bleeding Academic Research Consortium. Circulation. 2011;123:2736-47.

15. Bovill EG, Terrin ML, Stump DC, et al. Hemorrhagic events during therapy with recombinant tissue-type plasminogen activator, heparin, and aspirin for acute myocardial infarction. Results of the Thrombolysis in Myocardial Infarction (TIMI), Phase II Trial. Ann Intern Med. 1991;115:256-65.
16. Rubin DB. Inference and missing data. Biometrika 1976;63:581-92.

17. Marshall A, Altman DG, Holder RL, Royston P. Combining estimates of interest in prognostic modelling studies after multiple imputation: current practice and guidelines. BMC Med Res Methodol. 2009;9:57.

18. An international randomized trial comparing four thrombolytic strategies for acute myocardial infarction. The GUSTO investigators. N Engl J Med. 1993;329:673-82.

19. van Werkum JW, Heestermans AA, Zomer AC, et al. Predictors of coronary stent thrombosis: the Dutch Stent Thrombosis Registry. J Am Coll Cardiol. 2009;53:1399-409.

20. Dangas GD, Caixeta A, Mehran R, et al. Frequency and predictors of stent thrombosis after percutaneous coronary intervention in acute myocardial infarction. Circulation. 2011;123:1745-56.

21. Mehran R, Pocock S, Nikolsky E, et al. Impact of bleeding on mortality after percutaneous coronary intervention results from a patient-level pooled analysis of the REPLACE-2 (randomized evaluation of PCI linking angiomax to reduced clinical events), ACUITY (acute catheterization and urgent intervention triage strategy), and HORIZONS-AMI (harmonizing outcomes with revascularization and stents in acute myocardial infarction) trials. JACC Cardiovasc Interv. 2011;4:654-64.

22. Allison MA, Criqui MH, Wright CM. Patterns and risk factors for systemic calcified atherosclerosis. Arterioscler Thromb Vasc Biol. 2004;24:331-6.

23. Saw J, Bhatt DL, Moliterno DJ, et al. The influence of peripheral arterial disease on outcomes: a pooled analysis of mortality in eight large randomized percutaneous coronary intervention trials. J Am Coll Cardiol. 2006;48:1567-72.

24. White HD, Aylward PE, Gallo R, et al. Hematomas of at least $5 \mathrm{~cm}$ and outcomes in patients undergoing elective percutaneous coronary intervention: insights from the SafeTy and Efficacy of Enoxaparin in PCI patients, an internationaL randomized Evaluation (STEEPLE) trial. Am Heart J. 2010;159:110-6.

25. Ndrepepa G, Neumann FJ, Richardt G, et al. Prognostic value of access and non-access sites bleeding after percutaneous coronary intervention. Circ Cardiovasc Interv. 2013;6:354-61.

26. Dodson JA, Wang Y, Chaudhry SI, Curtis JP. Bleeding-avoidance strategies and outcomes in patients $\geq 80$ years of age with ST-elevation myocardial infarction undergoing primary percutaneous coronary intervention (from the NCDR CathPCI Registry). Am J Cardiol. 2012;110:1-6.

27. Wiviott SD, Braunwald E, McCabe $\mathrm{CH}$, et al. Prasugrel versus clopidogrel in patients with acute coronary syndromes. $\mathrm{N}$ Engl $\mathrm{J}$ Med. 2007;357:2001-15.

28. Wallentin L, Becker RC, Budaj A, et al. Ticagrelor versus clopidogrel in patients with acute coronary syndromes. N Engl J Med. 2009;361:1045-57.

29. Yetgin T, van der Linden MM, de Vries AG, et al. Adoption of prasugrel into routine practice: rationale and design of the Rijnmond Collective Cardiology Research (CCR) study in percutaneous coronary intervention for acute coronary syndromes. Neth Heart J. 2014;22:55-61. 